\title{
Recovering the full Navier Stokes equations with lattice Boltzmann schemes
}

\author{
François Dubois $^{a b}$, Benjamin Graille $^{a}$, Pierre Lallemand ${ }^{c}$ \\ ${ }^{a}$ Dpt. of Mathematics, University Paris-Sud, Bât. 425, F-91405 Orsay, France. \\ ${ }^{b}$ Conservatoire National des Arts et Métiers, LMSSC laboratory, F-75003 Paris, France. \\ ${ }^{c}$ Beijing Computational Science Research Center, Haidian District, Beijing 100094, China.
}

13 July 2016 赵

\begin{abstract}
We consider multi relaxation times lattice Boltzmann scheme with two particle distributions for the thermal Navier Stokes equations formulated with conservation of mass and momentum and dissipation of volumic entropy. Linear stability is taken into consideration to determine a coupling between two coefficients of dissipation. We present interesting numerical results for one-dimensional strong nonlinear acoustic waves with shocks.
\end{abstract}

\section{1) Introduction}

In this contribution, our program is to use the lattice Boltzmann schemes with multiresolution relaxation times [8] for the approximation of the full compressible Navier Stokes equations. Interesting results have been obtained previously in [11, 13] when using a single particle distribution. A popular idea proposed in [1, 10, 16, 17] is the use of several particle distributions. One particle distribution is devoted to the conservation of mass and momentum and an other one to the conservation of energy. This framework has been used in the context of Bhatnagar Gross Krook [2] approximation [6, 9, 14, 15]. The adaptation to the multiresolution relaxation times approach is not straightforward [4] and source terms must be added to a pure "collide-stream" algorithm in order to capture this nonlinear dissipation term.

In this contribution, we begin with elementary one-dimensional fluid flow with the D1Q3 multi relaxation times lattice Boltzmann scheme. The Taylor expansion method allows us to determine the underlying partial differential equations. We adapt this study to the case of only one advection-diffusion equation. The coupling of these two schemes for the approximation of the Navier Stokes equations conducts to difficulties. This defect has its origin in precise algebraic properties of lattice Boltzmann schemes and associated moments. We then admit to consider lattice Boltzmann schemes with source terms. We focus on a

* Published in November 2016 by the in American Institute of Physics Proceedings, volume 1786, 040003 (2016), doi.org/10.1063/1.4967541. Edition 20 November 2017. 
formulation of the Navier Stokes equations that takes into consideration the production of entropy. The coupling of two D1Q3 schemes is proposed and studied and first numerical results are presented.

\section{2) Fluid flow with a multi relaxation times D1Q3 lattice Boltzmann scheme}

We describe in this section a very simple but fundamental lattice Boltzmann scheme. We restric ourselves to one space dimension. The mesh is parameterized by a space step $\Delta x$. The nodes are located at the vertices $x=j \Delta x$, where $j$ is an integer. We suppose given three discrete velocities: $v_{0}=0, v_{+}=+1$ and $v_{-}=-1$. A first description of the degrees of freedom is associated to the density $f \equiv\left(f_{0}, f_{+}, f_{-}\right)$of particles. In the D1Q3 case, we have three kind of particles. The motionless particles $f_{0}(x, t)$, the particles $f_{+}(x, t)$ going from $x$ to $x+\Delta x$ during one time step $\Delta t$ and the particles $f_{-}(x, t)$ going from $x$ to $x-\Delta x$ during the time step. We adopt the so-called "acoustic scale" and the numerical velocity $\lambda \equiv \frac{\Delta x}{\Delta t}$ is supposed fixed for the entire contribution. From this distribution of particle densities, we construct two first moments: mass density

$$
\rho \equiv \rho_{0} \sum_{j=0,+,-} f_{j}=\rho_{0}\left(f_{+}+f_{-}+f_{0}\right)
$$

and momentum

$$
J \equiv \rho_{0} \lambda \sum_{j=0,+,-} v_{j} f_{j}=\rho_{0} \lambda\left(f_{+}-f_{-}\right) .
$$

The third momentum is the so-called "energy" defined by

$$
e \equiv \rho_{0} \lambda^{2}\left(f_{+}+f_{-}-2 f_{0}\right) \text {. }
$$

The second description of the degrees of freedom is due to the moments

$$
m \equiv(\rho, J, e) .
$$

An invertible matrix $M$ is therefore defined between the particles and the moments:

(1) $m \equiv\left(\begin{array}{c}\rho \\ J \\ e\end{array}\right)=M_{\mathrm{D} 1 \mathrm{Q} 3}\left(\begin{array}{c}f_{0} \\ f_{+} \\ f_{-}\end{array}\right) \equiv M_{\mathrm{D} 1 \mathrm{Q} 3} f, \quad M_{\mathrm{D} 1 \mathrm{Q} 3}=\rho_{0}\left(\begin{array}{ccc}1 & 1 & 1 \\ 0 & \lambda & -\lambda \\ -2 \lambda^{2} & \lambda^{2} & \lambda^{2}\end{array}\right)$.

The evolution algorithm is composed by two steps: relaxation and advection. In the relaxation step, density and momentum remain at equilibrium and do not change:

$$
\rho^{*}=\rho, J^{*}=J .
$$

At the contrary, the equilibrium energy is a given function of the two moments at equilibrium:

$$
e^{\mathrm{eq}}=e^{\mathrm{eq}}(\rho, J) \text {. }
$$

The energy after relaxation is obtained by a simple evolution:

$$
e^{*}=e+s_{e}\left(e^{\mathrm{eq}}-e\right) .
$$

The relaxation parameter $s_{e}$ must be chosen satisfying $0<s_{e}<2$ [12]. The particle distribution $f^{*}$ after relaxation is defined by

$$
f^{*}=M_{\mathrm{D} 1 \mathrm{Q} 3}^{-1}\left(\rho, J, e^{*}\right)^{\mathrm{t}} .
$$




\section{Recovering the full Navier Stokes equations with Lattice Boltzmann}

The advection step is a free displacement of the particles during one time step:

$$
f_{0}(x, t+\Delta t)=f_{0}^{*}(x, t), f_{+}(x, t+\Delta t)=f_{+}^{*}(x-\Delta x, t), f_{-}(x, t+\Delta t)=f_{-}^{*}(x+\Delta x, t) .
$$

\section{3) Taylor expansion method and associated algebraic tools}

We conduct the analysis of the multi relaxation times with the Taylor expansion method [3]. This approach is a kind of numerical Chapman-Enskog expansion with the space step $\Delta x$ as a small parameter. Equivalent partial differential equations emerge from this analysis for the conserved variables. We have for the previous example up to order 2 :

$$
\partial_{t} \rho+\partial_{x} J=\mathrm{O}\left(\Delta x^{2}\right), \quad \partial_{t} J+\partial_{x}\left(\frac{2}{3} \lambda^{2} \rho+\frac{1}{3} e^{\mathrm{eq}}\right)-\frac{1}{3} \sigma_{e} \Delta t \partial_{x} \theta_{e}=\mathrm{O}\left(\Delta x^{2}\right),
$$

with a coefficient $\sigma_{e}=\frac{1}{s_{e}}-\frac{1}{2}$ introduced by Hénon [7] and

$$
\theta_{e} \equiv \partial_{t} e^{\mathrm{eq}}+\lambda^{2} \partial_{x} J \simeq 3 \lambda^{2} \rho \partial_{x} u
$$

for the previous fluid D1Q3 scheme. We can compare these equations (2) with the unidimensional "isentropic" Navier Stokes equations

$$
\partial_{t} \rho+\partial_{x} J=0
$$

for mass conservation and

$$
\partial_{t} J+\partial_{x}\left(\rho u^{2}+p\right)-\partial_{x}\left(\rho \nu \partial_{x} u\right)=0
$$

for momentum conservation. Then the energy at equilibrium follows the relation

$$
e^{\mathrm{eq}}=3\left(\rho u^{2}+p\right)-2 \lambda^{2} \rho .
$$

The relaxation parameter is defined in (8) and is related to kinematic viscosity:

$$
\nu_{0}=\lambda \Delta x \sigma_{e} .
$$

In all generality, if the moments $m$ are related to the particle distribution $f$ via a relation of the type $m=M f$ with a invertible matrix $M$, we introduce [3] the momentum-velocity tensor $\Lambda$ according to

$$
\Lambda_{k \ell} \equiv \lambda \sum_{j} M_{k j} v_{j}\left(M^{-1}\right)_{j \ell} .
$$

For the previous D1Q3 model, with the ordering $\rho, J, e$ of the moments $m$, we have

$$
\Lambda_{\mathrm{D} 1 \mathrm{Q} 3}=\left(\begin{array}{ccc}
0 & 1 & 0 \\
\frac{2}{3} \lambda^{2} & 0 & \frac{1}{3} \\
0 & \lambda^{2} & 0
\end{array}\right) .
$$

The vector of conserved moments is denoted by $W$ and the defect of conservation $\theta_{\ell}$ is defined according to

$$
\theta_{\ell} \equiv \partial_{t} m_{\ell}^{\mathrm{eq}}+\sum_{p} \Lambda_{\ell p} \partial_{x} m_{p}^{\mathrm{eq}}
$$

for the non-conserved moments $m_{\ell}$. Then the $k^{\text {th }}$ conserved moment $W_{k}$ satisfies asymptotically the following second order partial differential equation

$$
\partial_{t} W_{k}+\sum_{\ell} \Lambda_{k \ell} \partial_{x} m_{\ell}^{\mathrm{eq}}-\Delta t \sum_{\ell} \sigma_{\ell} \Lambda_{k \ell} \partial_{x} \theta_{\ell}=\mathrm{O}\left(\Delta x^{2}\right) .
$$


with the Hénon coefficient [7] $\sigma_{\ell}$ defined in all generality according to

$$
\sigma_{\ell}=\frac{1}{s_{\ell}}-\frac{1}{2}
$$

The generalization to two and three dimensions is straightforward. We observe also that the equations (7) are always under a conservative form.

\section{4) Advection-diffusion with a multi relaxation times D1Q3 lattice Boltzmann scheme}

We modify the notations of the previous D1Q3 "MRT" scheme, replacing the particle distribution $f$ by the notation $g: g_{0}(x, t)$ is the motionless particles at $x$ during the time step $\Delta t$, $g_{+}(x, t)$ the density of particles going from $x$ to $x+\Delta x$ during one time step and $g_{-}(x, t)$ the density of particles going from $x$ to $x-\Delta x$ during $\Delta t$. The numerical velocity $\lambda$ remains fixed. We introduce three moments: a conserved variable $\zeta$, momentum $\psi$ and associated energy $\varepsilon$ in a way analogous to (1):

$$
\zeta \equiv \zeta_{0}\left(g_{0}+g_{+}+g_{-}\right), \psi \equiv \zeta_{0} \lambda\left(g_{+}-g_{-}\right), \varepsilon \equiv \zeta_{0} \lambda^{2}\left(g_{+}+g_{-}-2 g_{0}\right) .
$$

An invertible matrix $\widetilde{M}$ is defined by the condition

$$
(\zeta, \psi, \varepsilon)^{\mathrm{t}}=\widetilde{M}_{\mathrm{D} 1 \mathrm{Q} 3}\left(g_{0}, g_{+}, g_{-}\right)^{\mathrm{t}} .
$$

It is analogous to the matrix introduced in (1) except that the scaling factor $\rho_{0}$ is replaced by $\zeta_{0}$. For this model, only one moment is conserved and remains at equilibrium: $\zeta^{*}=\zeta$. The momentum $\psi$ and associated energy $\varepsilon$ at equilibrium are given functions of the scalar $\zeta$ :

$$
\psi^{\mathrm{eq}}=\psi^{\mathrm{eq}}(\zeta), \varepsilon^{\mathrm{eq}}=\varepsilon^{\mathrm{eq}}(\zeta) .
$$

The momentum and energy after relaxation $\psi^{*}$ and $\varepsilon^{*}$ are obtained by simple evolutions:

$$
\psi^{*}=\psi+s_{\psi}\left(\psi^{\mathrm{eq}}-\psi\right) \varepsilon^{*}=\varepsilon+s_{\varepsilon}\left(\varepsilon^{\mathrm{eq}}-\varepsilon\right)
$$

with $0<s_{\psi}<2$ and $0<s_{\varepsilon}<2$. The particle distribution $g^{*}$ after relaxation is obtained from the moments:

$$
\left(g_{0}^{*}, g_{+}^{*}, g_{-}^{*}\right)^{\mathrm{t}}=\left(\widetilde{M}_{\mathrm{D} 1 \mathrm{Q} 3}\right)^{-1}\left(\zeta, \psi^{*}, \varepsilon^{*}\right)^{\mathrm{t}} .
$$

The advection step is identical to the corresponding one for the fluid scheme:

$$
\left\{\begin{array}{l}
g_{0}(x, t+\Delta t)=g_{0}^{*}(x, t) \\
g_{+}(x, t+\Delta t)=g_{+}^{*}(x-\Delta x, t) \\
g_{-}(x, t+\Delta t)=g_{-}^{*}(x+\Delta x, t) .
\end{array}\right.
$$

We can analyze formally this scheme with the Taylor expansion method. With the matrix $\Lambda$ introduced in (5), the equivalent partial differential equation up to order 2 takes the form

$$
\partial_{t} \zeta+\partial_{x} \psi^{\mathrm{eq}}-\sigma_{\psi} \Delta t \partial_{x} \theta_{\psi}=\mathrm{O}\left(\Delta x^{2}\right)
$$

with

and

$$
\sigma_{\psi}=\frac{1}{s_{\psi}}-\frac{1}{2}
$$

$$
\theta_{\psi} \equiv \partial_{t} \psi^{\mathrm{eq}}+\partial_{x}\left(\frac{2}{3} \lambda^{2} \rho+\frac{1}{3} \varepsilon^{\mathrm{eq}}\right)
$$




\section{Recovering the full Navier Stokes equations with lattice Boltzmann}

This "thermal" lattice Boltzmann model is well adapted for the simulation of an advectiondiffusion equation of the type

$$
\partial_{t} \zeta+\partial_{x}\left(u_{0} \zeta\right)-\partial_{x}\left(\kappa \partial_{x} \zeta\right)=0
$$

The second order differential equation (9) simulated by the thermal lattice Boltzmann scheme is a good approximation to first order of (9) if the momentum at equilibrium $\psi^{\text {eq }}$ is given by

$$
\psi^{\mathrm{eq}}=u_{0} \zeta \text {. }
$$

The defect of equilibrium can be expanded:

$$
\theta_{\psi} \equiv\left(\frac{2}{3} \lambda^{2}-u_{0}^{2}\right) \partial_{x} \zeta+\frac{1}{3} \partial_{x} \varepsilon^{\mathrm{eq}}+\mathrm{O}(\Delta x)
$$

and the energy at equilibrium is proportional to the conserved variable:

$$
\varepsilon^{\mathrm{eq}}=\alpha \lambda^{2} \zeta \text {. }
$$

The identification of second order terms induces:

$$
\left(\frac{2+\alpha}{3} \lambda^{2}-u_{0}^{2}\right)\left(\frac{1}{s_{\psi}}-\frac{1}{2}\right) \Delta t=\kappa
$$

with a typical constraint for numerical stability: $-2<\alpha<1$. We take simply $s_{\varepsilon}=1.5$ for the third moment.

\section{5) Unidimensional Navier Stokes equations with energy conservation}

We try now to simulate the one dimensional Navier Stokes equations with the coupling the two previous models. The conserved variables are the volumic mass $\rho$, the momentum $J \equiv \rho u$, and the volumic total energy

$$
\rho E \equiv \rho\left(i+\frac{1}{2} u^{2}\right)
$$

obtained by adding the specific kinetic energy $\frac{1}{2} u^{2}$ to the internal one $i$. We suppose that the equation of state is a polytropic perfect gas with a ratio

$$
\gamma \equiv \frac{c_{p}}{c_{v}}
$$

of specific heats:

$$
p=(\gamma-1) \rho i=\rho r T .
$$

The expression of the sound velocity $c$ is classical:

$$
c^{2}=\gamma \frac{p}{\rho}=\gamma(\gamma-1) i=(\gamma-1) c_{p} T .
$$

The kinematic viscosity and thermal conductivity define the Prandtl number

$$
\operatorname{Pr}=\frac{\rho \nu c_{p}}{\kappa} \text {. }
$$

The conservation of mass, momentum and energy takes the form

$$
\left\{\begin{array}{l}
\partial_{t} \rho+\partial_{x} J=0 \\
\partial_{t} J+\partial_{x}\left(\rho u^{2}+p\right)-\partial_{x}\left(\rho \nu \partial_{x} u\right)=0 \\
\partial_{t}(\rho E)+\partial_{x}(\rho E u+p u)-\partial_{x}\left(\rho \nu u \partial_{x} u\right)-\partial_{x}\left(\kappa \partial_{x} T\right)=0 .
\end{array}\right.
$$


A first and natural idea proposed by Alexander et al. 11, Khobalatte and Perthame [10, McNamara and Alder [16], Shan and Chen [17] is to use two particle distributions. The first one $(f)$ devoted to the conservation of mass and momentum, the second one $(g)$ for the conservation of total energy. We consider this idea by a coupling of fluid and thermal D1Q3 lattice Boltzmann models. The conserved moments are those of the Navier-Stokes equations (10):

$$
\rho=\rho_{0} \sum_{j} f_{j}, J=\rho_{0} \lambda \sum_{j} v_{j} f_{j}, \rho E=\rho_{0} \lambda^{2} \sum_{j} g_{j}
$$

The nonconserved moments complete the set of moments for this double "D1Q3Q3":

$$
e=\rho_{0} \lambda^{2}\left(f_{+}+f_{-}-2 f_{0}\right), \psi=\rho_{0} \lambda^{3} \sum_{j} v_{j} g_{j}, \varepsilon=\rho_{0} \lambda^{4}\left(g_{+}+g_{-}-2 g_{0}\right) .
$$

The particle representation is now a vector with 6 components:

$$
f_{d}=\left(f_{0}, f_{+}, f_{-}, g_{0}, g_{+}, g_{-}\right)^{\mathrm{t}}
$$

and the vector of moments admits the expression

$$
m \equiv(\rho, J, \rho E, e, \psi, \varepsilon)^{\mathrm{t}} .
$$

We have $m=M f_{d}$ with a matrix $M$ that is, up to a permutation, the tensor product of the D1Q3 matrix with itself.

We focus now on previous works of Guo et al. [6, Li et al. [14, 15], Karlin et al. [9]) with the framework following strictly the approach of BKG [2]. These authors discretize with the lattice Boltzmann method the set of kinetic equations

$$
\partial_{t} f_{j}+v_{j} \bullet \nabla f_{j}=-\frac{1}{\tau_{f}}\left(f_{j}-f_{j}^{\mathrm{eq}}\right), \quad \partial_{t} g_{j}+v_{j} \bullet \nabla g_{j}=-\frac{1}{\tau_{g}}\left(g_{j}-g_{j}^{\mathrm{eq}}\right)+\frac{Z}{\tau_{f}}\left(f_{j}-f_{j}^{\mathrm{eq}}\right) .
$$

Remark that due to the $\frac{Z}{\tau_{f}}$ term in the right hand side of the second equation of $(11)$, the relaxation step is deeply transformed. We have adapted this idea for the multi relaxation times approach [4]. We distinguish three types of moments:

(i) the conserved moments $W$ such that $W_{k}^{*}=W_{k}$ for $k<N$,

(ii) the "first non-conserved" moments $m$ such that $m_{k}^{*}=\left(1-s_{k}\right) m_{k}+s_{k} m_{k}^{\text {eq }}$ and

(iii) the "second non-conserved" moments such that

$$
m_{\ell}^{*}=m_{\ell}-s_{\ell}\left(m_{\ell}-m_{\ell}^{\mathrm{eq}}\right)+K_{\ell k}\left(m_{k}-m_{k}^{\mathrm{eq}}\right),
$$

with $m_{k}$ in the first family of nonconserved moments. Our results [4] are not entirely satisfying and we have changed our point of view.

\section{6) Lattice Boltzmann algorithm for the simulation of the thermal Navier Stokes equations}

The important remark is that we can re-interpret the second equation of (11) as an usual advection of the particle distribution with a source term. In the following, we approach with a lattice Boltzmann scheme the full Navier Stokes equations as a system of conservative partial differential equations with a source term:

$$
\partial_{t} W+\partial_{x} F(W)-\partial_{x}(\Phi(W, \nabla W))=S .
$$




\section{Recovering the full Navier Stokes equations with lattice Boltzmann}

This choice is motivated by the fact that the equivalent equations of a lattice Boltzmann scheme are always conservative. In this contribution, we have chosen as "conserved variables" the mass $\rho$, the momentum $J \equiv \rho u$ and the volumic entropy. The volumic entropy $\zeta$ is the product of the volumic mass $\rho$ multiplied by the specific entropy $s$. Then $W=(\rho, J, \zeta)$. The equation of state is the one of a polytropic perfect gas

$$
p=(\gamma-1) \rho i=\rho r T=p_{0}\left(\frac{\rho}{\rho_{0}}\right)^{\gamma} \exp \left(\frac{\gamma\left(s-s_{0}\right)}{c_{p}}\right) .
$$

The sound velocity $c$ satisfies

$$
c^{2}=\gamma \frac{p}{\rho}=\gamma(\gamma-1) i
$$

as previously. With this $(\rho, J, \zeta)$ formulation, the Navier Stokes equations express the conservation of mass and momentum, and the production of entropy:

$$
\left\{\begin{array}{l}
\partial_{t} \rho+\partial_{x} J=0 \\
\partial_{t} J+\partial_{x}\left(\rho u^{2}+p\right)-\partial_{x}\left(\rho \nu \partial_{x} u\right)=0 \\
\partial_{t} \zeta+\partial_{x}(\zeta u)-\partial_{x}\left(\frac{\kappa}{T} \partial_{x} T\right)=\frac{\rho \nu}{T}\left(\partial_{x} u\right)^{2}+\frac{\kappa}{T^{2}}\left(\partial_{x} T\right)^{2}
\end{array}\right.
$$

We use two particle distributions $f$ and $g$ as previously. The first distribution $f$ devoted to mass and momentum and the second distribution $g$ to volumic entropy. The conserved moments are defined from the double particle distribution according to

$$
\rho=\rho_{0}\left(f_{0}+f_{+}+f_{-}\right), \quad J=\rho_{0} \lambda\left(f_{+}-f_{-}\right), \quad \zeta=\rho_{0} c_{p}\left(g_{0}+g_{+}+g_{-}\right) .
$$

The nonconserved moments are defined thanks to the previous considerations:

$$
e=\rho_{0} \lambda^{2}\left(f_{+}+f_{-}-2 f_{0}\right), \quad \psi=\rho_{0} c_{p} \lambda\left(g_{+}-g_{-}\right), \quad \varepsilon=\rho_{0} c_{p} \lambda^{2}\left(g_{+}+g_{-}-2 g_{0}\right) .
$$

We apply the general theory of multi relaxation times lattice Boltzmann schemes with the particle representation

$$
f_{d} \equiv\left(f_{0}, f_{+}, f_{-}, g_{0}, g_{+}, g_{-}\right)^{\mathrm{t}}
$$

and the momentum representation

$$
m \equiv(\rho, J, \rho E, e, \psi, \varepsilon)^{\mathrm{t}} .
$$

We have $m=M_{\mathrm{D} 1 \mathrm{Q} 3 \mathrm{Q} 3} f_{d}$. The matrix $M_{\mathrm{D} 1 \mathrm{Q} 3 \mathrm{Q} 3}$ between particles and moments is given by the relation

$$
M_{\mathrm{D} 1 \mathrm{Q} 3 \mathrm{Q} 3}=\rho_{0}\left(\begin{array}{cccccc}
1 & 1 & 1 & 0 & 0 & 0 \\
0 & \lambda & -\lambda & 0 & 0 & 0 \\
0 & 0 & 0 & c_{p} & c_{p} & c_{p} \\
-2 \lambda^{2} & \lambda^{2} & \lambda^{2} & 0 & 0 & 0 \\
0 & 0 & 0 & 0 & c_{p} \lambda & -c_{p} \lambda \\
0 & 0 & 0 & -2 c_{p} \lambda^{2} & c_{p} \lambda^{2} & c_{p} \lambda^{2}
\end{array}\right) .
$$

We must now specify the equilibrium functions for non-conserved moments:

$$
e^{\mathrm{eq}}=e^{\mathrm{eq}}(\rho, J, \zeta), \quad \psi^{\mathrm{eq}}=\psi^{\mathrm{eq}}(\rho, J, \zeta), \quad \varepsilon^{\mathrm{eq}}=\varepsilon^{\mathrm{eq}}(\rho, J, \zeta) .
$$

The coefficients $s_{e}, s_{\psi}$ and $s_{\varepsilon}$ for the relaxation of non-conserved moments, i.e.

$$
e^{*}=e+s_{e}\left(e^{\mathrm{eq}}-e\right), \quad \psi^{*}=\psi+s_{\psi}\left(\psi^{\mathrm{eq}}-\psi\right), \quad \varepsilon^{*}=\varepsilon+s_{\varepsilon}\left(\varepsilon^{\mathrm{eq}}-\varepsilon\right) .
$$

The time iteration of the D1Q3Q3 scheme can be simply written:

$$
\left\{\begin{array}{l}
f_{0}(x, t+\Delta t)=f_{0}^{*}(x, t), f_{ \pm}(x, t+\Delta t)=f_{ \pm}^{*}(x \mp \Delta x, t) \\
g_{0}(x, t+\Delta t)=g_{0}^{*}(x, t), g_{ \pm}(x, t+\Delta t)=g_{ \pm}^{*}(x \mp \Delta x, t) .
\end{array}\right.
$$


The adaptation to the presence of source terms is described in [5]. The gradients $\partial_{x} u$ and $\partial_{x} T$ are evaluated with second order centered finite differences.

\section{7) Linearized Navier Stokes equations}

An important step is the study of the linearized Navier Stokes equations. We consider a reference state $W_{0}=\left(\rho_{0}, \rho u_{0}, \rho s_{0}\right)$, with the associated sound velocity $c_{0}$ satisfying $c_{0}^{2}=\frac{\gamma p_{0}}{\rho_{0}}$. We linearize the Navier Stokes system (13) and obtain without difficulty

$$
\left\{\begin{array}{l}
\partial_{t} \rho+\partial_{x} J=0 \\
\partial_{t} J+\left(c_{0}^{2}-u_{0}^{2}-\frac{s_{0} c_{0}^{2}}{c_{p}}\right) \partial_{x} \rho+2 u_{0} \partial_{x} J+\frac{c_{0}^{2}}{c_{p}} \partial_{x} \zeta-\nu_{0} \partial_{x}^{2} \rho+\nu_{0} u_{0} \partial_{x}^{2} J=0 \\
\partial_{t} \zeta-u_{0} s_{0} \partial_{x} \rho+s_{0} \partial_{x} J+u_{0} \partial_{x} \zeta-\frac{\nu_{0}}{\operatorname{Pr}}\left((\gamma-1) c_{p}-\gamma s_{0}\right) \partial_{x}^{2} \rho-\gamma \frac{\nu_{0}}{\operatorname{Pr}} \partial_{x}^{2} \zeta=0 .
\end{array}\right.
$$

We first decouple and simplify the system 20. We obtain after this operation a simple system:

$$
\left\{\begin{array}{l}
\partial_{t} \rho+\partial_{x} J=0 \\
\partial_{t} J+\left(c_{0}^{2}-u_{0}^{2}\right) \partial_{x} \rho+2 u_{0} \partial_{x} J-\nu_{0} \partial_{x}^{2} \rho+\nu_{0} u_{0} \partial_{x}^{2} J=0 \\
\partial_{t} \zeta+u_{0} \partial_{x} \zeta-\gamma \frac{\nu_{0}}{\operatorname{Pr}} \partial_{x}^{2} \zeta=0
\end{array}\right.
$$

The system of equations (21) is nothing else than the juxtaposition of a linearized version of the system (2) of fluid equations and an advection-diffusion equation of the type (9). Then it is easy to derive an equation for the equilibrium moment:

$$
\frac{2}{3} \lambda^{2} \rho+\frac{1}{3} e^{\mathrm{eq}}=\left(c_{0}^{2}-u_{0}^{2}\right) \rho+2 u_{0} J
$$

The defect of conservation $\theta_{e}$ can be evaluated in a pure algebraic way:

$$
\theta_{e}=3\left(\lambda^{2}-3 u_{0}^{2}-c_{0}^{2}\right) \partial_{x} J-6 u_{0}\left(c_{0}^{2}-u_{0}^{2}\right) \partial_{x} \rho \simeq 3 \lambda^{2} \partial_{x} J .
$$

The coefficient of relaxation $s_{e}$ is determined through the kinematic viscosity: $\nu_{0}=\sigma_{e} \lambda \Delta x$. From the scalar "thermal" equation, we deduce an expression for the equilibrium moment $\psi^{\mathrm{eq}}=u_{0} \zeta$. For the defect of conservation $\theta_{\psi}$, we have

$$
\theta_{\psi}=\left(\frac{2}{3} \lambda^{2}-u_{0}^{2}\right) \partial_{x} \zeta+\frac{1}{3} \partial_{x} \varepsilon^{\mathrm{eq}} .
$$

The compatibility at second order is provided under the condition

$$
\left[\left(\frac{2}{3} \lambda^{2}-u_{0}^{2}\right) \zeta+\frac{1}{3} \varepsilon^{\mathrm{eq}}\right] \sigma_{\psi} \Delta t=\gamma \frac{\nu_{0}}{\operatorname{Pr}} \zeta
$$

Then the equilibrium of the moment $\varepsilon$ is easy to determine:

$$
\varepsilon^{\mathrm{eq}}=3\left(\frac{\gamma}{\operatorname{Pr}} \frac{\sigma_{e}}{\sigma_{\psi}}-\frac{2}{3}+\frac{u_{0}^{2}}{\lambda^{2}}\right) \lambda^{2} \zeta \equiv \alpha \lambda^{2} \zeta
$$

In order to enforce stability $(-2<\alpha<1)$, we suggest the following link between two relaxations:

$$
\sigma_{\psi}=\frac{3}{2} \frac{\gamma}{\operatorname{Pr}} \sigma_{e}
$$

We consider again the linearized Navier Stokes equations introduced in (20). We maintain the previous relations for relaxation coefficients: $\nu_{0}=\sigma_{e} \lambda \Delta x, \sigma_{\varepsilon}=1.5$ and the relation 


\section{Recovering the full Navier Stokes equations with LATtice Boltzmann}

(23). To assume compatibility between the equations $(7)$ and $(20)$, the equilibria $e^{\text {eq }}, \psi^{\text {eq }}$ and $\varepsilon^{\mathrm{eq}}$ are necessarily the following linear functions of the conserved variables $\rho, J$ and $\zeta$ :

$$
\left\{\begin{aligned}
e^{\mathrm{eq}} & =\left(3\left(1-\frac{s_{0}}{c_{p}}\right) c_{0}^{2}-3 u_{0}^{2}-2 \lambda^{2}\right) \rho+6 u_{0} J+3 \frac{u_{0}^{2}}{c_{p}} \zeta \\
\psi^{\mathrm{eq}} & =-u_{0} s_{0} \rho+s_{0} J+u_{0} \zeta \\
\varepsilon^{\mathrm{eq}} & =\left(-3\left(s_{0} c_{0}\right)^{2}-6 s_{0} u_{0}^{2}+3 s_{0} c_{0}^{2}+2-2 s_{0}-\frac{2}{\gamma}\right) \rho \\
& +6 u_{0} s_{0} J+3\left(c_{p} u_{0}^{2}+s_{0} c_{0}^{2}\right) \zeta .
\end{aligned}\right.
$$

For the simulation of a simple linear wave with the following parameters:

$$
\left\{\begin{array}{l}
\gamma=1.4, \operatorname{Pr}=1, c_{0}=\frac{\lambda}{2}, u_{0}=0, s_{0}=0, \\
s_{e}=1.9, \nu=6.57910^{-4}, \Delta x=\frac{1}{40}, T_{f}=120 \Delta t,
\end{array}\right.
$$

we have observed numerical stability. This fundamental property was also realized with the important modification of the parameters: $u_{0}=0.15 \lambda$ and $s_{0}=0.2 c_{p}$.

\section{8) D1Q3Q3 lattice Boltzmann scheme for the volumic entropy Navier Stokes equations}

The matrix $M_{\mathrm{D} 1 \mathrm{Q} 3 \mathrm{Q} 3}$ between particles and moments is still given by the relation (16). The equilibrium of non-conserved moments (17) is parameterized by nonlinear functions. We must also specify the coefficients $s_{e}, s_{\psi}$ and $s_{\varepsilon}$ for the relaxation (18) of the non-conserved moments. The discrete time iteration of the D1Q3Q3 scheme follows the relations (19). The Taylor expansion method at first order proposes partial differential equations satisfied by the conserved variables $W=(\rho, J, \zeta \equiv \rho s)$ :

$$
\partial_{t} W_{k}+\Lambda_{k \ell} \partial_{x} m_{\ell}^{\mathrm{eq}}=\mathrm{O}(\Delta t)
$$

with a momentum-velocity tensor $\Lambda$ given in (4). We have for this D1Q3Q3 lattice Boltzmann scheme

$$
\Lambda=\left(\begin{array}{cccccc}
0 & 1 & 0 & 0 & 0 & 0 \\
\frac{2 \lambda^{2}}{3} & 0 & 0 & \frac{1}{3} & 0 & 0 \\
0 & 0 & 0 & 0 & 1 & 0 \\
0 & \lambda^{2} & 0 & 0 & 0 & 0 \\
0 & 0 & \frac{2 \lambda^{2}}{3} & 0 & 0 & \frac{1}{3} \\
0 & 0 & 0 & 0 & \lambda^{2} & 0
\end{array}\right)
$$

The equivalent equations at first order take the form

$$
\partial_{t} \rho+\partial_{x} J=\mathrm{O}(\Delta x), \quad \partial_{t} J+\partial_{x}\left(\frac{2 \lambda^{2}}{3} \rho+\frac{1}{3} e^{\mathrm{eq}}\right)=\mathrm{O}(\Delta x), \quad \partial_{t} \zeta+\partial_{x} \psi^{\mathrm{eq}}=\mathrm{O}(\Delta x)
$$

They are compared to the Navier stokes equations of gas dynamics (13). By identification of first order terms, the "energy" at equilibrium $e^{\text {eq }}$ is given by the relation (3) and

$$
\psi^{\mathrm{eq}}=\zeta u \text {. }
$$

Thus two equilibria for nonequilibrium moments are fixed.

For the second order analysis, the Hénon's coefficients [7] are defined according to

$$
\sigma_{e}=\frac{1}{s_{e}}-\frac{1}{2}, \quad \sigma_{\psi}=\frac{1}{s_{\psi}}-\frac{1}{2}, \quad \sigma_{\varepsilon}=\frac{1}{s_{\varepsilon}}-\frac{1}{2} .
$$




\section{Franç ois Dubois, Benjamin Graille and Pierre Lallemand}

The defects of conservation (6) have to be estimated before the determination of the second order equivalent partial differential equations (7). For the present D1Q3Q3 lattice Boltzmann scheme, we have

$$
\left\{\begin{array}{l}
\partial_{t} \rho+\partial_{x} J=\mathrm{O}(\Delta t) \\
\partial_{t} J+\partial_{x}\left(\rho u^{2}+p\right)=\frac{\Delta t}{3} \sigma_{e} \partial_{x} \theta_{e}+\mathrm{O}\left(\Delta x^{2}\right) \\
\partial_{t} \zeta+\partial_{x}(\zeta u)=\Delta t \sigma_{\psi} \partial_{x} \theta_{\psi}+\mathrm{O}\left(\Delta x^{2}\right) .
\end{array}\right.
$$

The first defect of conservation

$$
\theta_{e} \equiv \partial_{t} e^{\mathrm{eq}}+\lambda^{2} \partial_{x} J
$$

can be approximated by $3 \lambda^{2} \rho \partial_{x} u$. Then the relation

$$
\nu=\sigma_{e} \lambda \Delta x
$$

determines the relaxation coefficient $s_{e}$ thanks to (28). For the second defect of conservation

$$
\theta_{\psi} \equiv \partial_{t} \psi^{\mathrm{eq}}+\frac{2}{3} \lambda^{2} \partial_{x} \zeta+\frac{1}{3} \partial_{x} \varepsilon^{\mathrm{eq}}
$$

we have, after integration by parts and using the first order equations (26),

$$
\theta_{\psi}=p \partial_{x} s+\partial_{x}\left[\frac{1}{3}\left(2 \lambda^{2} \zeta+\varepsilon^{\mathrm{eq}}\right)-\left(\left(\rho u^{2}+p\right) s\right)\right] .
$$

This expression is approximated by $\partial_{x}\left[\frac{1}{3}\left(2 \lambda^{2} \zeta+\varepsilon^{\mathrm{eq}}\right)-\left(\left(\rho u^{2}+p\right) s\right)\right]$. The entropy equation (third equation of (13)) is compared with the third equation of (29):

$$
\partial_{t} \zeta+\partial_{x}(\zeta u)-\partial_{x}^{2}\left\{\sigma_{\psi} \Delta t\left[\frac{1}{3}\left(2 \lambda^{2} \zeta+\varepsilon^{\mathrm{eq}}\right)-\left(\left(\rho u^{2}+p\right) s\right)\right]\right\}=\mathrm{O}\left(\Delta x^{2}\right) .
$$

Then

$$
\sigma_{\psi} \Delta t\left[\frac{1}{3}\left(2 \lambda^{2} \zeta+\varepsilon^{\mathrm{eq}}\right)-\left(\left(\rho u^{2}+p\right) s\right)\right]=\kappa \log \left(\frac{T}{T_{0}}\right) .
$$

With the relations $(23)$ and (30), we obtain for the third momentum for entropy

$$
\varepsilon^{\mathrm{eq}}=\frac{2 \rho c_{p} \lambda^{2}}{\gamma} \log \left(\frac{T}{T_{0}}\right)+\left[3\left(\rho u^{2}+p\right)-2 \lambda^{2} \rho\right] s .
$$

The relation (32) is clearly non-trivial. It is an interesting property of the present scheme to have determined this algebraic relation in the full nonlinear framework. For the polytropic perfect gas, the pressure is given by (12) and the relation (32) becomes

$$
\varepsilon^{\mathrm{eq}}=2 \lambda^{2}\left[\rho\left(s-s_{0}\right)+\left(1-\frac{1}{\gamma}\right) c_{p} \rho \log \left(\frac{\rho}{\rho_{0}}\right)\right]+\left[3\left(\rho u^{2}+p\right)-2 \lambda^{2} \rho\right] s .
$$

The present D1Q3Q3 algorithm can be implemented in the following way:

(i) consider the particle distribution $f_{d} \equiv\left(f_{0}, f_{+}, f_{-}, g_{0}, g_{+}, g_{-}\right)^{\mathrm{t}}$,

(ii) compute the six moments $m \equiv(\rho, J, \zeta, e, \psi, \varepsilon)^{\mathrm{t}}$, by $m=M_{D 1 Q 3 Q 3} f_{d}$ with $M_{D 1 Q 3 Q 3}$ defined by the relation (16),

(iii) fix the relaxation coefficients $s_{e}, s_{\psi}, s_{\varepsilon}$ of the non-conserved moments $e, \psi, \varepsilon$ through the associated $\sigma^{\prime} s$ thanks to 28 with $\sigma_{e}$ determined by $(30), \sigma_{\psi}$ according to (23) and $s_{\varepsilon}=1.5$,

(iv) determine the moments $e^{\mathrm{eq}}, \psi^{\mathrm{eq}}, \varepsilon^{\mathrm{eq}}$ at equilibrium with the relations (3), (27) and (32) respectively,

(v) compute the moments $e^{*}, \psi^{*}, \varepsilon^{*}$ after relaxation with the relations (18), 


\section{Recovering the full Navier Stokes equations with Lattice Boltzmann}

(vi) compute the particle distribution $f_{d}^{*}$ after relaxation thanks to

$$
f_{d}^{*}=\left(M_{D 1 Q 3 Q 3}\right)^{-1}\left(\rho, J, \zeta, e^{*}, \psi^{*}, \varepsilon^{*}\right)^{\mathrm{t}},
$$

(vii) evaluate the gradients $\partial_{x} u$ and $\partial_{x} T$ with second order centered finite differences, add a source term to the momentum $\zeta$ as proposed by the third equation in (13) and the algorithm described in [5],

(viii) iterate the scheme in time according to 19 .

\section{9) First numerical experiments}
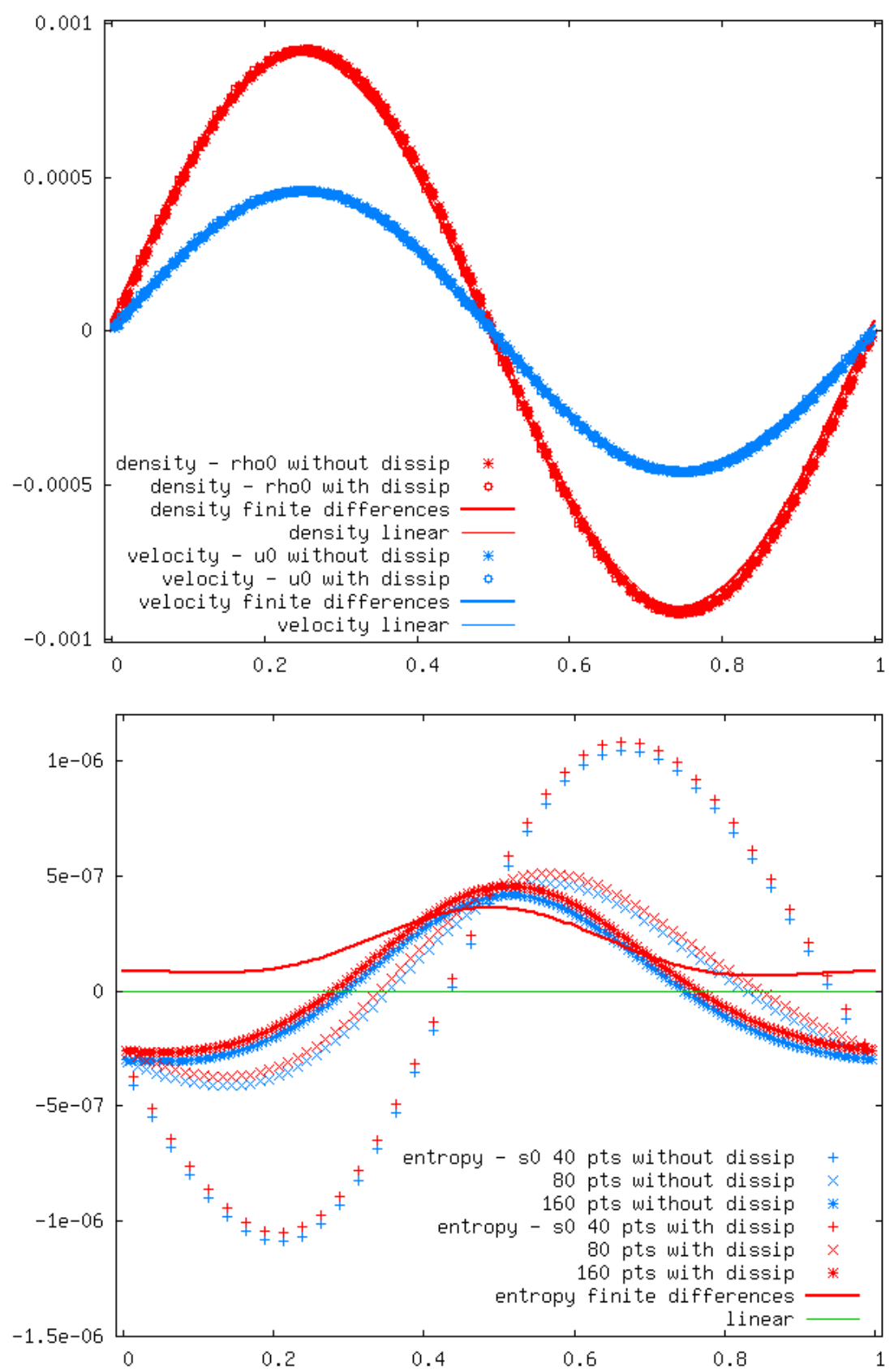

Figure 1. Progressive linear wave, periodic boundary conditions, $\delta \rho=0.001 \rho_{0}$. Density and velocity (top). Entropy (bottom). 

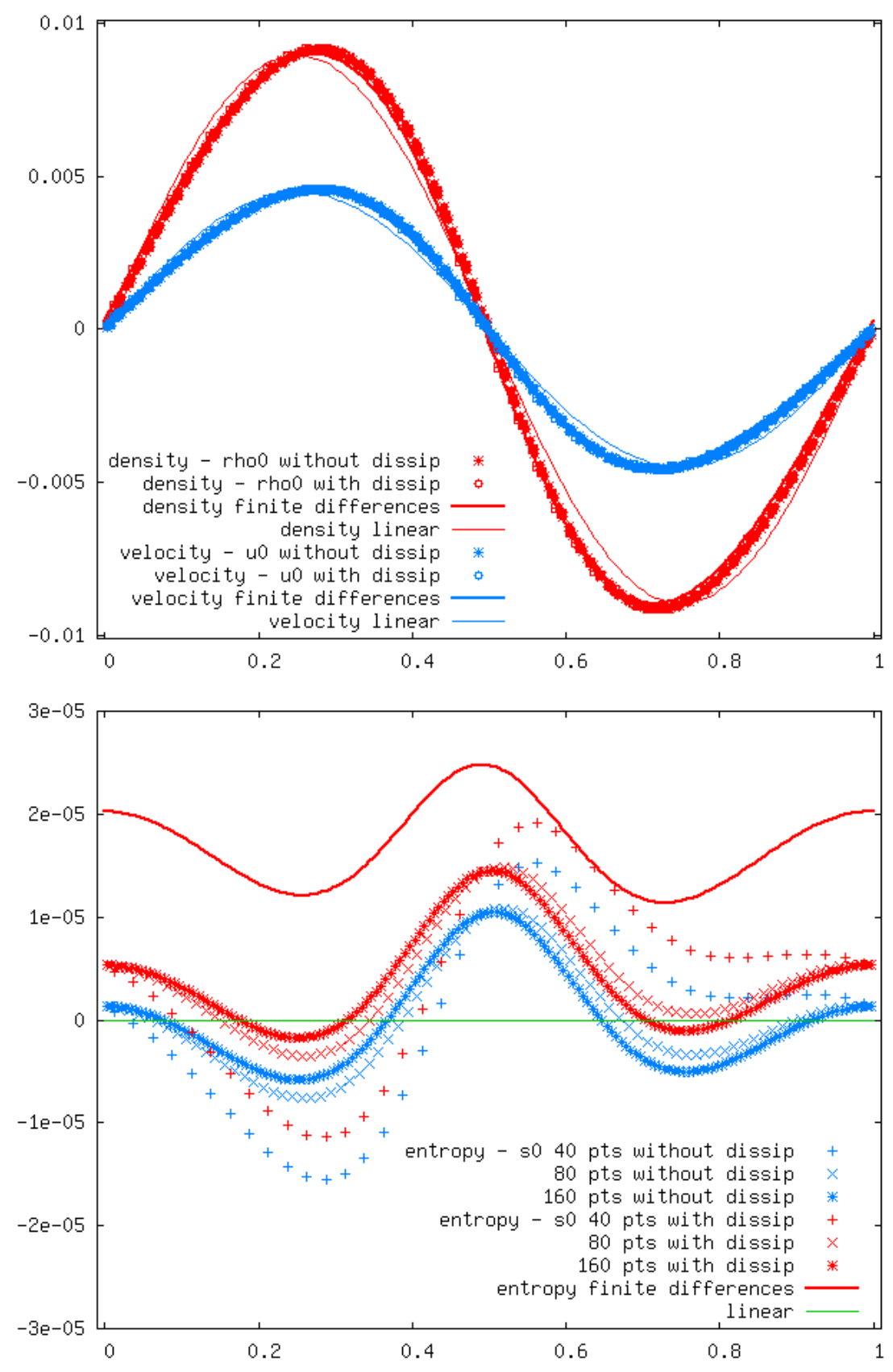

Figure 2. Progressive nonlinear wave, periodic boundary conditions, $\delta \rho=0.01 \rho_{0}$. Density and velocity (top). Entropy (bottom).

We have used the algorithm described in the previous section for the simulation of periodic waves. The general parameters are

$$
\gamma=1.4, \operatorname{Pr}=1, c_{0}=\frac{\lambda}{2}, s_{0}=0, T_{\max }=3, \nu=6.57910^{-4} .
$$

We have used three meshes with $\Delta x=\frac{1}{40}, \frac{1}{80}, \frac{1}{160}$. We present three numerical experiments. The initial condition is a simple acoustic wave with an initial variation $\delta \rho$ of density. For the first experiment (see Figure 1), the result is essentially described by the linearized equations. The variation of entropy is infinitesimal. 

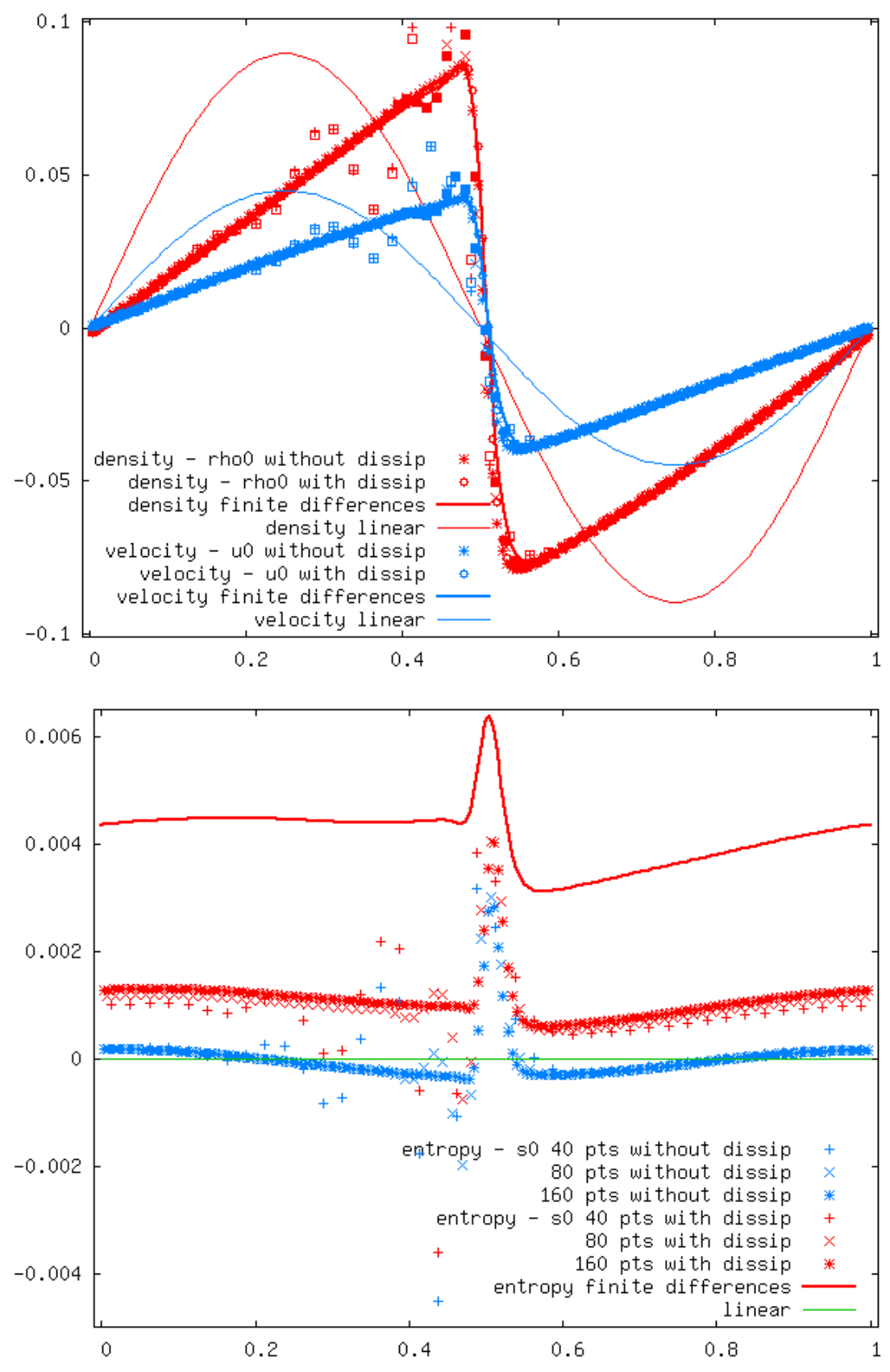

Figure 3. Strong nonlinear wave, periodic boundary conditions, $\delta \rho=0.1 \rho_{0}$. Density and velocity (top). Entropy (bottom).

With the second experiment described in Figure 2, nonlinear effects are clearly visible for the density and velocity results. In order to validate these effects, we have developed a finite difference software based on the formulation (13) and using second order centered finite differences and explicit first order time integration. The variation of entropy is very small and is of good quality. With $\delta \rho=\frac{\rho_{0}}{100}$, a shock wave is generated (see Figure 3). The production of entropy is essentially localized in the region of high variation of the fields. On Figure 4, we present the transient evolution of specific entropy and specific energy. Without the dissipation source term of (13), the total energy if not perfectly conserved and the specific 
entropy is quasi constant. With this source term, the defect for total energy is very small and the production of entropy remains moderate in comparison to our finite difference simulator. The extension of the previous work to two and three space dimensions is the next step of this work, typically with the coupled lattice Boltzmann schemes D2Q9-D2Q5 and D3Q19-D3Q7.

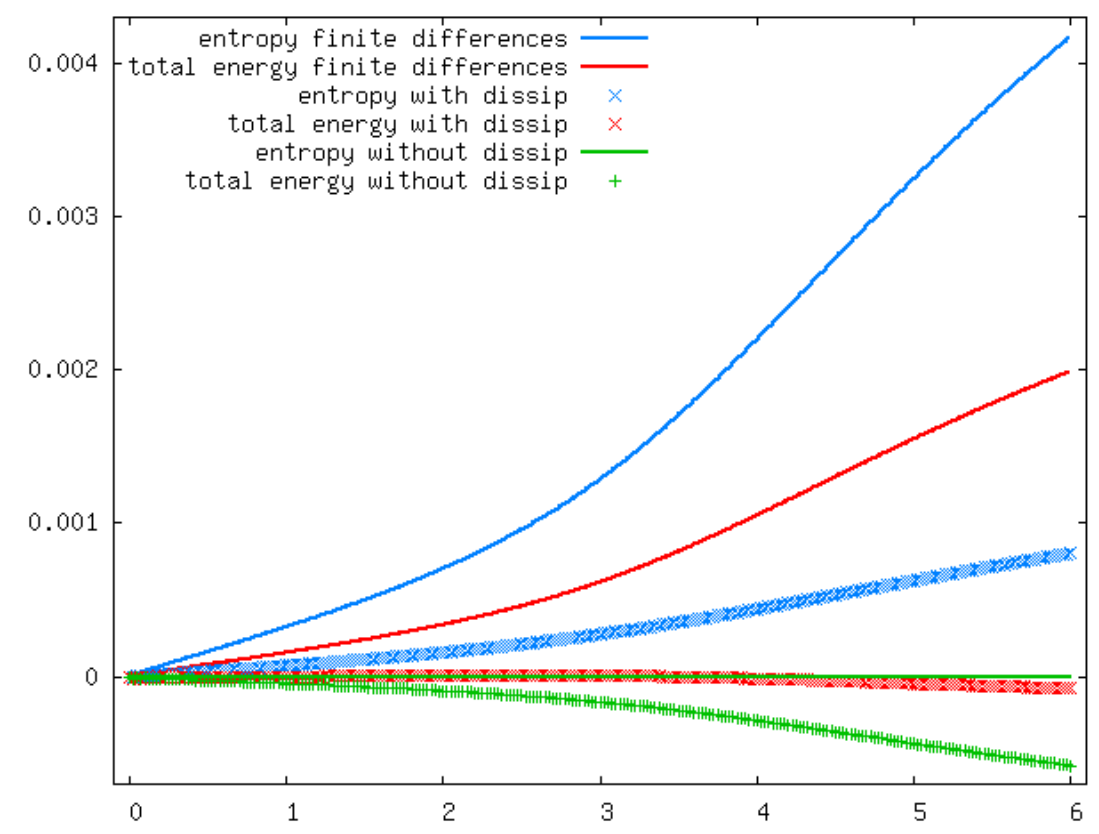

Figure 4. Strong nonlinear wave $\left(\delta \rho=0.1 \rho_{0}\right)$. Time evolution of entropy and total energy.

\section{Acknowledgements}

This work is supported by the French "Climb" Oseo project. A part of this work has been realized during the stay of two of us at the Beijing Computational Science Research Center. We thank the colleagues of CSRC for their hospitality. Last but not least, the authors thank the referee who suggested several points in need of improvement.

\section{References}

[1] F. Alexander, S. Chen and J. Sterling, "Lattice Boltzmann thermohydrodynamic", Physical Review E, 47, R2249-R2252 (1993).

[2] P. Bhatnagar, E. Gross and M. Krook, "A Model for Collision Processes in Gases. I. Small Amplitude Processes in Charged and Neutral One-Component Systems", Physical Review, 94, 511-525, 1954.

[3] F. Dubois, "Equivalent partial differential equations of a lattice Boltzmann scheme", Computers and Mathematics with Applications, 55, 1441-1449 (2008).

[4] F. Dubois, "Liens entre des schémas de Boltzmann sur réseau et les équations de Navier Stokes thermiques", Seminar, Institut Henri Poincaré, Paris, February 2016. 


\section{Recovering the full Navier Stokes equations with LATtice Boltzmann}

[5] F. Dubois, P. Lallemand, M.M. Tekitek, "Taylor expansion method for linear lattice Boltzmann schemes with an external drift. Application to boundary conditions", Springer Lecture Notes in Computational Science and Engineering", 99, R. Abgrall et al. Editors, 89-107 (2014).

[6] Z. Guo, C. Zheng and B. Shi, "Thermal lattice Boltzmann equation for low Mach number flows: decoupling model", Physical Review E, 75, 036704 (2007).

[7] M. Hénon, "Viscosity of a Lattice Gas", Complex Systems, 1, 763-789 (1987).

[8] D. D'Humières, "Generalized Lattice-Boltzmann Equations", in: AIAA Rarefied Gas Dynamics: Theory and Applications, Progress in Astronautics and Aeronautics, 159, AIAA, Washington, D.C., 450-458 (1992).

[9] I.V. Karlin, D. Sichau and S.S. Chikatamarla, "Consistent two-population lattice Boltzmann model for thermal flows", Physical Review E, 88, 063310 (2013).

[10] B. Khobalatte and B. Perthame. "Maximum principle on the entropy and second-order kinetic schemes", Mathematics of Computation, 62, 119-131, 1994.

[11] P. Lallemand and F. Dubois, "Comparison of Simulations of Convective Flows", Communications in Computational Physics, 17, 1169-1184 (2015).

[12] P. Lallemand and L.S. Luo, Theory of the lattice Boltzmann method: Dispersion, dissipation, isotropy, Galilean invariance, and stability, Physical Review E, 61, 6546-6562, (2000).

[13] P. Lallemand and L.S. Luo, "Theory of the lattice Boltzmann method: Acoustic and thermal properties in two and three dimensions", Physical Review E, 68, 036706 (2003).

[14] Q. Li, Y.L. He, Y. Wang and W.Q. Tao, "Coupled double-distribution-function lattice Boltzmann method for the compressible Navier-Stokes equations", Physical Review E, 76, 056705 (2007).

[15] Q. Li, K.H. Luo, Y.L. He, Y.J. Gao and W.Q. Tao, "Coupling lattice Boltzmann model for simulation of thermal flows on standard lattices", Physical Review E, 85, 016710, (2012).

[16] G. McNamara and B. Alder, "Analysis of the lattice Boltzmann treatment of hydrodynamics", Physica A, 194, 218-228 (1993).

[17] X. Shan and H. Chen, "Lattice Boltzmann model for simulating flows with multiple phases and components", Physical Review E, 47, 1815-1819 (1993). 